\title{
Discussion: how can we improve diagnosis of dentin hypersensitivity in the dental office?
}

\author{
Jens C. Türp
}

Received: 1 February 2012 /Accepted: 23 November 2012/Published online: 28 December 2012

(C) The Author(s) 2012. This article is published with open access at Springerlink.com

Keywords Dentin hypersensitivity · Diagnosis of DHS · Dentinal pain

\section{What is known?}

Dentin hypersensitivity (DHS) is characterized by a sharp, short-lasting "dentinal" pain originating from pulpal tissues in a healthy pulp as a consequence of an external stimulus, which can be thermal (hot; cold, including air), electrical, mechanical, osmotic (sweet; sour), or chemical [19].

Its occurrence and intensity among individuals lies within a clinical spectrum that ranges from occasional stimulusreliant moderate pain to frequent stimulus-dependent intense pain.

\section{What are the problems?}

A search in reveals that the number of publications related to the diagnosis of DHS is limited (Table 1). This may be an indication that the issue of making a diagnosis is either an easy or a difficult task. In fact, the latter is the case.

Time is needed to make a correct diagnosis because (a) a thorough patient history is required and (b) DHS is a diagnosis of exclusion: it is confirmed only after possible other conditions have been diagnostically eliminated.

Unfortunately, a validated screening checklist of DHSrelated predisposing, initiating, and perpetuating risk factors identified in clinical or epidemiological studies is not yet available.

\section{J. C. Türp $(\bowtie)$}

Clinic for Reconstructive Dentistry and Temporomandibular Disorders, Dental School, University of Basel, Basel, Switzerland e-mail: jens.tuerp@unibas.ch
Since individuals may be affected by DHS in varying degree, mild forms may not be reported by the patient to the dentist. Conversely, in other patients, DHS may substantially impair oral health-related quality of life (OHRQoL), for instance during drinking, eating, and oral hygiene [5]. Not every patient who suffers from DHS may know where to seek help to alleviate the pain.

\section{What are the recommendations for daily practice?}

1. In every (new) patient, irrespective of a patient complaint of DHS, a verbal screening is recommended, during which she/he is asked the following questions:

(a) Do your teeth hurt when eating or drinking hot, cold, or acidic food or drinks?

(b) Do your teeth hurt when you brush your teeth?

If patients answer with "yes" on at least one of these questions, specific pain characteristics should be recorded (e.g., character, severity, site, onset, etc.).

2. Clinicians may ask or look for:

- Personal behavior (e.g., consumption of highly acidic drinks or food; overzealous dental hygiene);

- Previous dental procedures (e.g., scaling and other periodontal therapy; tooth bleaching; restorative procedures);

- Clinical signs (e.g., dental erosion; gingival recession; exposed cervical dentin; periodontitis; caries; tooth fractures).

3. In patients with suspected DHS (due to positive findings in step 1 and, possibly, step 2), a thorough differential diagnosis is indispensable. Hence, other forms of orofacial pain, including pulpitis, periodontal pain, cracked tooth syndrome, and atypical odontalgia, must be excluded, before the diagnosis of DHS is made. 
Table 1 The results of a PubMed search reveal that many more publications focus on the therapy of dentin (hyper)sensitivity than on diagnostic aspects. Search date December 10, 2012

\begin{tabular}{ll}
\hline Search strategy & Hits \\
\hline ("Dentin Sensitivity"[Mesh]) and "therapy" [Subheading] & 1,348 \\
("Dentin Sensitivity"[Mesh] and "diagnosis" [Subheading]) & 381 \\
\hline
\end{tabular}

4. A specific DHS-related clinical examination is obligatory in cases with positive findings in steps 1 and, possibly, 2, and negative findings in step 3:

It is suggested to move a blunt exploratory probe in the mesiodistal (or distomesial) direction on the exposed dentin $[12,15]$. In addition, a jet of air should be directed towards the affected tooth region $[12,15]$. These tactile and thermal stimuli should provoke the DHS-associated pain.

Pain intensity should be measured by using an 11-point numerical rating scale, a $100-\mathrm{mm}$ visual analog scale, or a validated graphic pain scale, such as the Faces Pain Scale [6].

Pain quality should be characterized by verbal descriptors ("pain adjectives"), either according to the patient's spontaneous report or by the use of a validated questionnaire $[10,11]$.

5. Since DHS may affect OHRQoL, it is recommended to include this pain-related dimension during the patient assessment. A suitable instrument for this purpose is the Oral Health Impact Profile [17], which needs to be completed by the patient. In addition to the original version of this questionnaire, validated translations are available in other languages, including Arabic [1], Chinese [21], Croatian [13], Dutch [20], French [2], German [8], Hungarian [18], Japanese [7, 22], Portuguese [14], Russian [4], Slovenian [16], Spanish [9], and Turkish [3].

6. Finally, education of the public should be fostered to ensure that individuals affected by and suffering from DHS know that dental practitioners may be able to alleviate their symptoms.

Open Access This article is distributed under the terms of the Creative Commons Attribution License which permits any use, distribution, and reproduction in any medium, provided the original author(s) and the source are credited.

\section{References}

1. Al-Jundi MA, Szentpétery A, John MT (2007) An Arabic version of the Oral Health Impact Profile: translation and psychometric properties. Int Dent J 57:84-92
2. Allison P, Locker D, Jokovic A et al (1999) A cross-cultural study of oral health values. J Dent Res 78:643-649

3. Baran I, Nalcaci R (2011) Self-reported problems before and after prosthodontic treatments according to newly created Turkish version of Oral Health Impact Profile. Arch Gerontol Geriatr 53:e99 e105

4. Barer GM, Gurevich KG, Smirniagina VV et al (2007) [Validation of Oral Health Impact Profile (OHIP) quality of life questionnaire in Russian patients with evidence of chronic generalized periodontitis]. Stomatologiia (Mosk) 86:27-30

5. Bekes K, John MT, Schaller HG et al (2009) Oral health-related quality of life in patients seeking care for dentin hypersensitivity. J Oral Rehabil 36:45-51

6. Hicks CL, von Baeyer CL, Spafford PA et al (2001) The faces pain scale - revised: toward a common metric in pediatric pain measurement. Pain 93:173-183

7. Ide R, Yamamoto R, Mizoue T (2006) The Japanese version of the Oral Health Impact Profile (OHIP) - validation among young and middle-aged adults. Community Dent Health 23:158-163

8. John MT, Patrick DL, Slade GD (2002) The German version of the Oral Health Impact Profile - translation and psychometric properties. Eur J Oral Sci 110:425-433

9. Lopez R, Baelum V (2006) Spanish version of the Oral Health Impact Profile (OHIP-Sp). BMC Oral Health 6:11

10. Melzack R (1975) The McGill Pain Questionnaire: major properties and scoring methods. Pain 1:277-299

11. Melzack R (1987) The short-form McGill pain questionnaire. Pain 30:191-197

12. Miglani S, Aggarwal V, Ahuja B (2010) Dentin hypersensitivity: recent trends in management. J Conserv Dent 13:218-224

13. Petricevic N, Celebic A, Papic M et al (2009) The Croatian version of the Oral Health Impact Profile questionnaire. Coll Antropol 33:841-847

14. Pires CP, Ferraz MB, de Abreu MH (2006) Translation into Brazilian Portuguese, cultural adaptation and validation of the Oral Health Impact Profile (OHIP-49). Braz Oral Res 20:263-268

15. Porto ICCM, Andrade AKM, Montes MAJR (2009) Diagnosis and treatment of dentinal hypersensitivity. J Oral Sci 51:323332

16. Rener-Sitar K, Celebic A, Petricevic N et al (2009) The Slovenian version of the Oral Health Impact Profile questionnaire (OHIPSVN): translation and psychometric properties. Coll Antropol 33:1177-1183

17. Slade GD, Spencer AJ (1994) Development and evaluation of the Oral Health Impact Profile. Community Dent Health 11:3-11

18. Szentpétery A, Szabo G, Marada G et al (2006) The Hungarian version of the Oral Health Impact Profile. Eur J Oral Sci 114:197203

19. Türp JC, Hugger A, Löst C et al (2009) Vorschlag einer Klassifikation der Odontalgien. Schmerz 23:448-460

20. van der Meulen MJ, John MT, Naeije M et al (2008) The Dutch version of the Oral Health Impact Profile (OHIP-NL): translation, reliability and construct validity. BMC Oral Health 8:11

21. Wong MC, Lo EC, McMillan AS (2002) Validation of a Chinese version of the Oral Health Impact Profile (OHIP). Community Dent Oral Epidemiol 30:423-430

22. Yamazaki M, Inukai $M, B a b a ~ K$ et al (2007) Japanese version of the Oral Health Impact Profile (OHIP-J). J Oral Rehabil 34:159168 